\title{
Design of Motor Control System Based on Wireless Sensor Network
}

\author{
https://doi.org/10.3991/ijoe.v14i10.9311
}

Herong Yuan $(\bowtie)$

Nanjing Polytechnic Institute, Nanjing, China

147922013 @q. com

Jingbin Song

Changzhou Vocational Institute of Mechatronic Technology, Changzhou, China

\begin{abstract}
More and more modern devices are controlled by miniature special motors. But many existing miniature special motors are using mostly independent test methods through wire communication. In this paper, starting from the requirements of motor testing and the situation of workshop environment, a motor measurement and control system based on wireless sensor network is proposed and designed. This paper elaborates the relevant technical analysis and specific implementation of TT \& C module, wireless terminal, wireless router, wireless gateway and host computer measurement and control module. At last, the test function and effect of the system are explained according to the test samples. The reliability and stability of the system are verified through reliability analysis.
\end{abstract}

Keywords - motor control system, wireless sensor network, electrical measurement and control, wireless communication

\section{Introduction}

Along with the development of the national economy, various electrical equipment have gradually become an indispensable part of our life. People require more comfort and automation control of the automobiles as well. Many of the devices in traditional electrical equipment are mechanically controlled, adjusted by manual regulation, such as motor rape, automatic antenna, lacquer washing, water tank cooling fan and so on. Accordingly, its automation level and equipment stability are not high. Besides, it is easy to damage and difficult to maintain, resulting in poor user experience. In contrast, modern electrical equipment usually use miniature special motors, some of which are used as actuators and others for positioning. By applying the embedded technology to the control of the mechanical structure, the automatic control of the equipment can be realized, it greatly improves the automation control and user experience of the equipment [1-2]. At present, there are many workshops producing miniature special motors, and their production and testing equipment are mostly semi-automated test equipment. 
In the workshop production line, the motor control link is adopted to detect the quality of motor. The testing equipment is mostly semi-automatic equipment. They are usually independent and comprehensive testing fixtures, mainly including motor test fixtures and PC [3]. The motor test fixtures has the function of motor control and project detection, and the test results are sent to the computer software of the PC by wired communication. Besides, the power supply and communication line of the equipment are connected by cable, which requires that the detection workshop should plan the equipment position and cable direction in advance. At the same time, when the motor is detected, each device needs special test workers' operation, and each test table is independent. Then the administrator will merge and report the motor detection data stored in different PC machines. The traditional micro motor detection equipment is not suitable for additional and mobile testing equipment because of the need to arrange cable. As each test fixtures needs a PC, the utilization rate of the PC machine is low, the equipment is large and the test operation is more complex. In addition, each device needs personnel participation in the test process, increasing the cost of testing manpower. Meanwhile, the test data of different equipment need to be artificially integrated, which is not conducive to management. Gradually, the equipment cable is easy to damage, and equipment fault inquiry and maintenance are more complicated.

Wireless communication is one of the fastest developing technologies in the field of communication in recent years. It mainly uses the characteristics that electromagnetic waves can radiate and propagate in space, and it is used as the carrier of information to exchange data in space [4].

Wireless sensor network (WSN) is composed of a large number of cheap sensor nodes in the monitoring area. Due to the communication and computing power of sensor nodes, they communicate with each other and form a multi hop intelligent motor control system covering the monitoring area [5]. The wireless sensor network has the characteristics of low power, low cost, self-organization. Therefore, the dynamic reconfiguration of the system network is very strong, it means that the topology of the network will not be changed by the single node failure, the new node joining or the movement of the node. The wireless sensor network is a dynamic network based on data. Nodes in the sensor network can select the unique identity number or random assignment number of the whole network according to the user's needs. Because of the random deployment of nodes in the network, there is no inevitable connection between the node number and the location, so users generally query the information in the network according to the data itself, which is closer to the natural language communication habit. At the same time, each node of the wireless sensor network has the function of data integrity checking and authentication in the process of communication. It uses AES encryption algorithm to ensure the security of data transmission, and its communication link has a strong reliability. The above characteristics make WSN have great application value and market demand in many fields such as environmental monitoring, medical care, military field, target tracking and so on. At present, the application of wireless sensor networks has penetrated into the fields of smart home and industrial control, in which building automation and industrial motor control systems are the main direction of application [6]. 
The industrial wireless network is a kind of wireless sensor network which is equipped with equipment control, data acquisition and other functions for industrial field. It uses wireless communication technology to subtract the cables needed for communication in the cable industrial network, making the system easy to use, maintain and expand. In the cable industry network, the typical cable installation cost is between 130 and 650 dollars per meter. If the wireless communication mode is adopted, the installation cost of the cable can be reduced by $20-80 \%$ [7-8]. Actually, industrial wireless network has a broad application prospect because of its own characteristics, including saving the cost of controlling network, reducing the complexity of equipment installation, improving the extensibility and maintainability of the equipment, reducing the limitation of the space area of the system and so on. The emergence of industrial wireless network enriches the way of information transmission in the field of industrial control. It is a revolutionary technology to reduce the cost of system design, to improve the category of industrial application and system maintainability.

According to the complexity of testing items, workshops can be divided into two kinds of testing methods. One is for complex situations of equipment and environment, such as high temperature resistance, high voltage resistance, high frequency noise interference, torque measurement and control, etc. In this case, the testing items are usually independent, and each item is detected by pipeline. After the current project is qualified, it will be transferred to the next item. In this way, the detection equipment has high pertinence, precision and reliability, but the detection efficiency and space utilization ratio are relatively low. The other one is for testing items such as coil current, speed, angle detection and out of step detection. In general, comprehensive test fixtures are usually used for testing.

The traditional integrated test fixtures are used to detect the requirements of the testing items, taking the micro stepper motor of vehicle as an example, which mainly involves the detection of the coil current, running speed and out of step detection. The traditional integrated test fixture does not contain torque measurement and control device, so it can not accurately measure the performance of the motor with load, and the detection method of stepper motor's out-of-step problem is still in the experimental stage.

\section{Overall Plan of System}

\subsection{System measurement and control technology}

The system measurement and control technology mainly includes four parts: the micro stepper motor measurement and control system, the simulated load loading technology, the torque detection technology and the angle detection technology.

Micro stepper motor measurement and control technology mainly involves micro stepper motor drive, coil current detection, speed detection and other technologies.

Driving technology of stepper motor. Although the stepper motor is a numerical control element, the driving current can't drive the stepper motor to work normally because of the weak digital pulse signal. Therefore, the driving circuit must be added 
between the input interface of the motor and the output interface of the digital pulse signal source. The design of the drive circuit must be combined with the technical parameters of the stepper motor, there are three parameters that are closely related to drive circuit design, which are briefly described as follows:

- Unloaded starting frequency:The pulse frequency which enables the stepper motor to start normally under unloaded condition is called unloaded starting frequency. When the frequency of the driving pulse is higher than the unloaded starting frequency, the motor may not start normally and howl.

- Inherent step angle of motor: The inherent step angle of the motor refers to the angle that the driver sends a pulse signal to make the stepper motor turn over. In actual work, the step angle is related to the specific design of the driver.

- The phase number of the stepper motor: Usually the stepper motor has different number of coils. Currently, there are two phase, three phase, four phase and five phase stepper motors commonly used. The stepper motor has different coil phases, so the step angle is different.

The torque frequency characteristics of several typical stepper motors are shown in Figure 1, and the output torque of the stepper motor, driven by the high frequency rate pulse, is rapidly decreasing. In practical applications, a high frequency drive pulse is often needed to drive the step to make the motor run steadily at the specified rate. If the driving pulse is directly controlled by the driving pulse at the starting frequency, the step motor is easily lost and even can't be started at all. When the stepper motor starts with load, the starting frequency of the stepper motor is lower than that of the unloaded condition, which is more likely to cause the motor to lose step and block the rotor. After the stepper motor completes the normal operation task, if the control driver stops sending the driving pulse at a certain moment, it is easy to cause the overshoot of the motor because of the inertia of the motor rotor or the load.

The frequency drive mode is usually used to improve the stability of the stepper motor in carrying and running at high speed, and the typical pulse frequency control curve is shown in Figure 2. The frequency control of the stepper motor is to start with a driving pulse lower than the starting frequency, and then increase the driving frequency to the operating frequency according to a certain rule. In the same way, the driving frequency is reduced in accordance with a certain rule in the deceleration to avoid the out of step or overshoot [9-10].

Coil current detection technology. The typical method of coil current detection is to select the appropriate precision resistance and series in the drive loop, and then use the current sampling, signal conversion and other peripheral current to detect the current of the sampling resistance. At present, there are a variety of current sampling chips on the market. These chips can detect the micro current signals of different ranges through the extra capacitance and resistance and so on. The output mode is usually the current signal, which can be converted from series resistance to voltage signal. Besides, a suitable $\mathrm{A} / \mathrm{D}$ converter is used to collect and process the voltage signal so as to get the current information. The accuracy of the above method depends on the resistance value of the sampling resistance and the accuracy of the A/D converter. The smaller high precision resistance is usually selected and the thermal effect of the resistance is 
taken into full consideration. Thus the detection method has the advantages of low detection cost and simple structure.

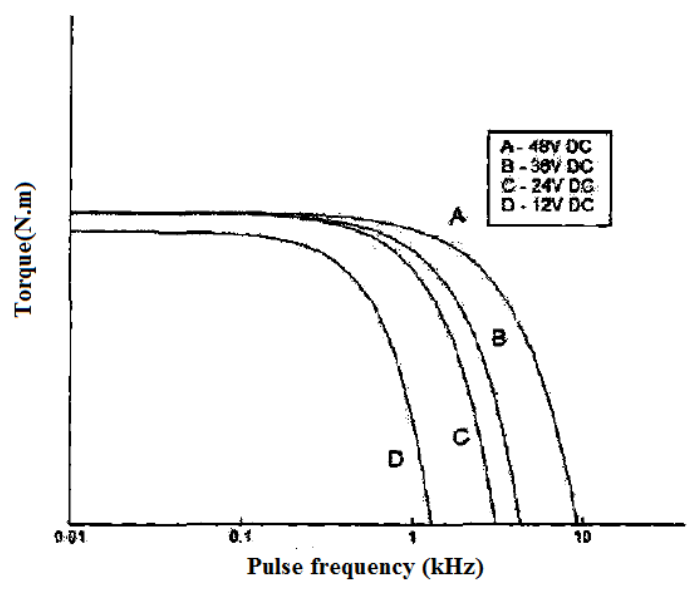

Fig. 1. Torque-Frequency characteristic of typical stepper motor

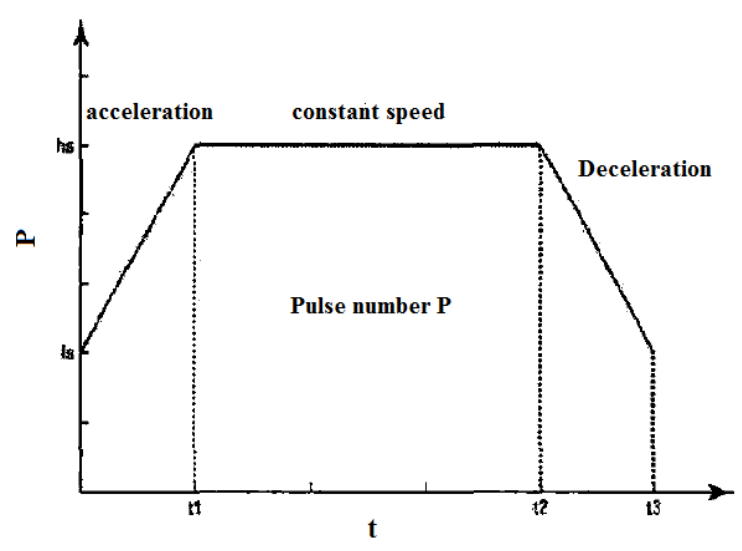

Fig. 2. Lifting frequency pulse frequency curve

Speed detection technology. There are two ways of speed detection: one is to use special sensor measurements, such as speed generator and photoelectric encoder. The rotating body of the sensor is usually connected with the motor, and the speed information is obtained by the output signal of the sensor. The other way is to use the angle sensor to detect the angle of the motor. The speed of the motor can be obtained by calculating the angle of the motor running in the unit time. Actually, the first sensor speed measurement method needs to increase the interface between the microcontroller and the sensor, its measurement accuracy and anti-interference ability is strong. The accuracy of the second methods depends on the precise use of the timer and the accuracy of the angle sensor. It is suitable for occasions with low accuracy and low speed operation. 


\subsection{ZigBee wireless network}

The wireless network composed of ZigBee technology is a wireless personal area network (LR-WPAN) with low rate, low loss and flexible throughput. The most basic unit of the network is equipment. According to the size of the device function, it can be divided into FFD and RFD. FFD devices can communicate with multiple RFD devices or FFD devices at the same time, but RFD devices can only communicate with one FFD device. There are three types of device in ZigBee wireless network: coordinator, router and terminal device. The primary task of the coordinator is to establish the wireless network and manage the network equipment, and in the process of networking, it has the function of distributing network address and receiving wireless data. The router is responsible for discovering the network, which has the functions of managing the network equipment, maintaining the information of the routing table, and forwarding the data. Usually, the terminal equipment has the function of discovering the network and receiving the wireless data. In fact, each device in the ZigBee network has a unique 64 bit IEEE address, and the coordinator assigns a short address of 16 bits for each device. The device can communicate with a 64 bit IEEE address or a 16 bit short address in the network. Since each network in multiple ZigBee networks has a uniquely identified network address (PANID), the device can communicate in different ZigBee networks based on PANID and 16 bit short addresses [11].

ZigBee wireless network topology is divided into three structures: star, tree and mesh, the characteristics of each of them are as follows.

- Star topology: The star topology is characterized by the presence of coordinator as the only network management device in the network, and the topology is shown in Figure 3. The coordinator is a full function device (FFD), which is responsible for the creation, maintenance and management of the network. The other devices can choose FFD or reduced function device (RFD) as terminals, but it is important to note that the terminal devices only have network and data transceiver functions, and do not participate in network management. Since the communication between any two uncoordinator devices in a star topology must be forwarded by the coordinator, the structure is more suitable for small area communication, such as home automation, medical care and so on.

- Tree topology: The tree topology contains router devices and only one coordinator device, whose topology is shown in Figure 4. The tree topology is centered around the coordinator device and scatters around the router device. For the establishment of tree topology network, the coordinator first selects a network address (PANID) to set up the network, and sends the beacon detection to the surrounding devices with its own short address as 0 . After receiving the beacon, the device in the coordination range applies and joins the network to form the first cluster of the tree topology network. When router devices are found and added to the network, the coordinator assigns an address block to the router, including a number of 16 bit short addresses, then the router configures and broadcasts its own beacon, at the same time, it allowing other devices to join the network through the router to form the next cluster. More specifically, when a terminal device is added to a coordinator or router, the coordinator or router assigns 16 bit short addresses to the terminal device [12]. As a 
contrast, in the tree network topology, the router devices can be added to the network by the same type of device to form a multilevel tree cluster network structure, making the radio network radiation wider than the star network topology, so it is especially suitable to be used in large distribution areas.

- Mesh topology: Similarly, there are coordinators, routers and terminal devices in mesh topology, whose topology is shown in Figure 5. Compared with the tree topology network, any two devices in mesh topology can communicate with each other in the wireless radiation range, so the network structure has strong variability and does not need to be determined in the establishment of the network. At the same time, the coordinator needs to be able to maintain network management and routers, so its storage capacity, computing power, complexity, and cost requirements are higher compared to other topologies [13]. As a result, the network topology has strong network ability and coverage. Therefore, it is more suitable for occasions with wide distribution of device and high networking capability, such as industrial control, intelligent agriculture and so on.

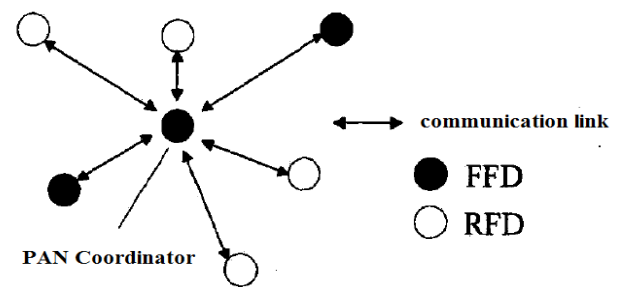

Fig. 3. Structure of star topology

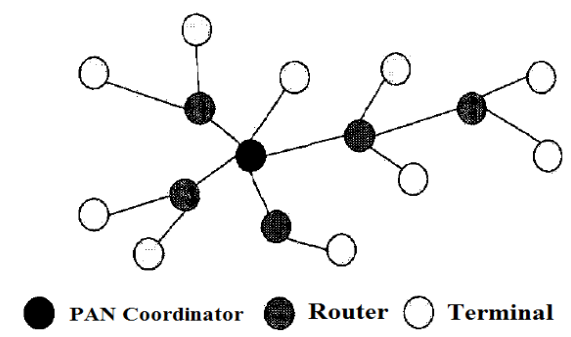

Fig. 4. Structure of tree topology

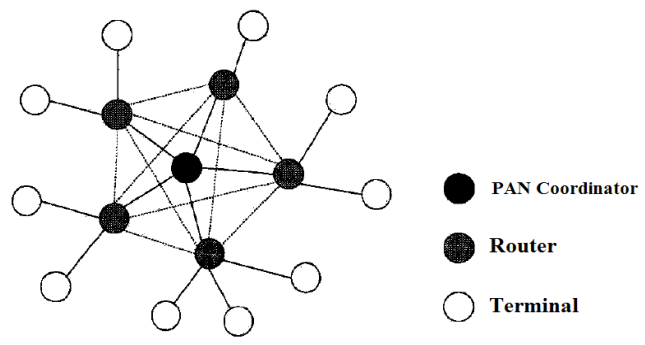

Fig. 5. Structure of mesh topology 


\subsection{System design scheme}

The system designed in this paper is mainly applied to the quality detection link in the motor production line of electronics workshops. According to the needs of the step motor detection in the workshop, the following important factors should be considered.

1. System generality. Because of the diversity of micro stepping motor products for workshop production, different types of motor are usually different in motor shape, motor drive voltage, driving frequency and other parameters. In order to improve the utilization ratio of the equipment, the testing system will be compatible with the testing requirements of different specifications of motor products.

\section{Testing items and technical indicators}

- Loading and testing of simulated load. The system can provide continuous and controllable torque on the rotating shaft of the motor to realize the function demand of analog load and plugging. The loading range of torque is $0 \sim 300 \mathrm{~N} . \mathrm{cm}$ and the precision is $1 \mathrm{~N} . \mathrm{cm}$. It can detect and display the motor load in real time, and its detection accuracy is higher than that of the simulated load device.

- Angle detection. The system has the function of angle detection, which can locate and measure the rotation angle of the motor shaft, and the precision of angle detection is 0.1

- Current detection. The system has the motor coil current detection function, which can display the current in the form of graphic curve and detect the current peak, plug current and other information, further, the precision of current detection is $1 \mathrm{~mA}$.

- Speed detection. The system has the function of motor speed detection, which can display the speed information of the motor with load.

3. Necessary function. The necessary functions include configuration of the motor detection item and the product information, the out-of-step detection, the configuration of the product's factory status, the edited product label interface, the display and storage of the test results.

4. Reliability of system. The system has high reliability, it can work for more than 24 hours in a single time, and the detection speed less than 30s/ per station. Additionally, the sample detection through the measurement and control equipment has good repeatability and reproducibility.

5. (Other requirements. In addition to meeting the functional requirements and reliable operation of system, the following requirements should be met as far as possible: first, reduce the complexity of system installation and maintenance; second, improve the utilization of the old equipment, the utilization of workshop space and the extensibility of the equipment; third, improve the efficiency of the workshop and save the human resources; finally, provide the compatibility of the database with the workshop management system, and realize the sharing of the test data.

According to the functional requirements of the system, the measurement and control system of step motor based on wireless sensor network is put forward in combination with the current detection equipment and the environment of the workshop. [14] 
The control strategy of decentralized detection and centralized management is adopted to integrate the detection data and improve the detection efficiency, in this way, the data sharing of the detection data and the workshop management system can be realized. At the same time, the use of wireless communication technology instead of the partial wired cable avoids the complexity of the workshop wiring and improves the scalability and mobility of the equipment.

As shown in Figure 6, the overall structure of the system includes three parts: PC, wireless communication module and measurement and control module. Specifically, PC is mainly used for the control of test and control commands and the storage and display of test results. The wireless communication module is mainly used for the wireless transmission of control commands and data. Through the analysis of the environment, functional requirements and system complexity of production lines, the system selects ZigBee wireless communication technology and adopts ZigBee tree network topology, including wireless terminal, wireless routing and wireless gateway, where ZigBee wireless terminal uses the serial communication mode to exchange data with the measurement and control module, and the ZigBee wireless gateway uses the USB serial communication mode to exchange data with PC. Further, the measurement and control module is mainly used to construct the measurement and control platform and detect the performance of the micro stepping motor, including the motor drive module, the current detection module, the torque measurement module, the angle detection module, the serial communication module and the power module.

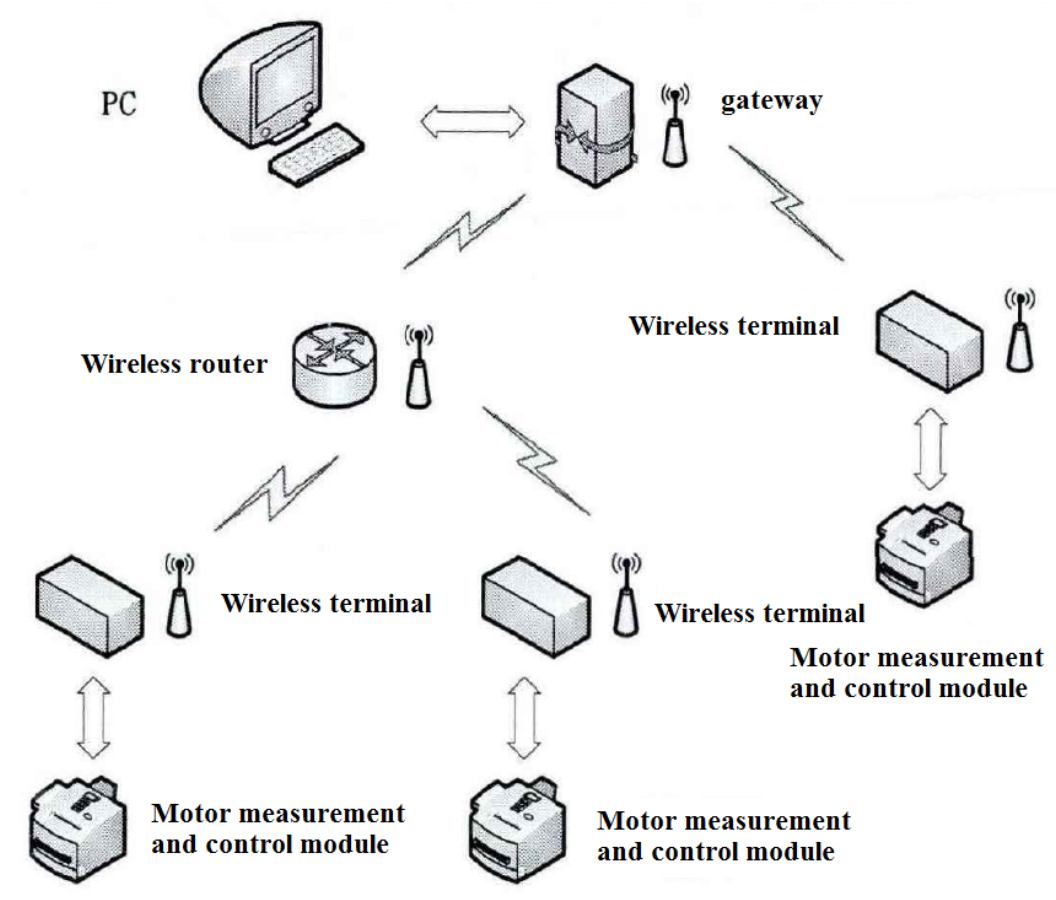

Fig. 6. Structure of the system 
The operating principle of the system is as follows:

Firstly, ZigBee coordinator establishes the wireless network, the terminal and router device search and join the wireless network. Secondly, the system control module sends the ready state confirmation information to PC through the terminal equipment. After $\mathrm{PC}$ receives the ready state confirmation information, it sends and executes the measurement and control commands. Thirdly, after receiving the command and control command of PC, the system begins to measure the stepper motor and sends the measurement and control information to PC via the terminal device, and then PC will display and store the results of measurement and control. At last, after testing a product, PC verifies the test data and sends qualified or unqualified information to the measurement and control module. The measurement and control module receives the qualified information and automatically begins to detect the next product; while the unqualified information is received, the test procedure for unqualified products is carried out according to the requirements of the workshop.

\section{$3 \quad$ System Testing and Analysis}

The operation and testing of the system are mainly explained by the setting, operation results and reliability analysis of the stepper motor's measurement and control. The relevant parameters of the motor measurement and control system are shown in Table 1.

Table 1. Stepper motor parameters

\begin{tabular}{|l|c|}
\hline \multicolumn{1}{|c|}{ Item } & Description \\
\hline Working voltage & $9-16 \mathrm{~V}$ \\
\hline Rated voltage & $12 \mathrm{~V}$ \\
\hline Rated frequency & $180 \mathrm{PPS}$ \\
\hline Rated speed & $1.65 \mathrm{rpm}$ \\
\hline Rated torque & $135 \mathrm{~N} . \mathrm{cm}$ \\
\hline Starting torque & $\geq 70 \mathrm{~N} . \mathrm{cm}$ \\
\hline Maximum torque & $<250 \mathrm{~N} . \mathrm{cm}$ \\
\hline Locking torque & $\geq 70 \mathrm{~N} . \mathrm{cm}$ \\
\hline Cyclic step number & 5400 \\
\hline Electric current & $\leq \mathbf{2 5 0 m A}$ \\
\hline
\end{tabular}

According to Table 1 , the step motor is driven by the pulse of $12 \mathrm{~V}$ voltage and $200 \mathrm{~Hz}$ frequency, every 7200 driving pulses make the motor rotated for one cycle. The rated load of 135 N.cm may be applied, running current is less than $250 \mathrm{~mA}$, and rated speed is $1.65 \mathrm{rpm}$.

The completed system is mainly divided into four parts: mechanical equipment, motor general tester, wireless communication module and host computer. When the device is installed, the test terminal, wireless router and gateway are set up in the same network, and the device addresses are set to a non-repeating address. The power of each 
device is turned on, then the wireless device is waiting for networking. When networking is successful, the measurement and control parameters of the motor can be set.

The test results show that the actual running position of the test out-of-step is $44.92^{\circ}$, actual end angle is $134.78^{\circ}$. The motor operates under the pulse drive of voltage $12 \mathrm{~V}$ and frequency $200 \mathrm{HZ}$, and there is no out-of-step phenomenon. The motor's speed, current, locked torque and locked rotor current are all within the testing standard under the condition of load.

The measurement and control system of the step motor based on wireless network is applied to the workshop production line. The reliability of its measurement and control function is closely related to the quality of the factory products. The main contents of the system reliability test are the reliability of the current detection device, the reliability of the motor measurement and control device, the reliability of wireless communication, the repeatability and the stability of the system.

The reliability of wireless communication is analyzed through the packet loss situation of wireless communication and the interference of wireless communication by the scene environment. In practice, wireless communication technology has a certain delay compared with wire communication. When there is a large area of metal obstacles in the scene environment, the communication quality will decline. The system adopts ZigBee application scheme with external antenna for wireless communication. Compared with the wireless devices without the power amplifier module, the designed wireless devices have strong communication ability and high communication quality. It can make up the defects of the wireless network radiation range, and meet the communication needs of the workshop.

\section{Conclusions}

With the rapid increase of application requirements of stepper motors, stepper motor measurement and control system has become an important guarantee for the quality of stepper motors and the production efficiency of workshops. Aiming at the defects of the traditional stepper motor measurement and control system, a motor measurement and control system based on wireless sensor network is designed and implemented in this paper. The overall scheme of the system is designed by combining wireless network technology with traditional stepper motor measurement and control system. The system uses the ZigBee technology to construct the communication network of the distributed measurement and control system. It selects the ZigBee tree network topology, and uses the serial communication technology to realize the communication between the wireless terminal and the motor measurement and control module. When the system design is completed, the stability of the system is tested. The experimental results show that the system has high detection accuracy, and it can run steadily for a long time. 


\section{$5 \quad$ Acknowledgments}

This work is sponsored in part by QingLan Project in Jiangsu Province.

\section{References}

[1] Harwit, E. (2001). The Impact of WTO Membership on the Automobile Industry in China. China Quarterly, 167(167): 655-670. https://doi.org/10.1017/S0009443901000365

[2] Liu S. J. (2002). Tendency of Globalization of Automobile Industry and Its Impact on China's Automobile Industry. China Industrial Economy, 6: 20-21.

[3] Kim, W., Shin, D., Chung, C. C. (2012). The Lyapunov-based controller with a passive nonlinear observer to improve position tracking performance of microstepping in permanent magnet stepper motors. Automatica, 48(12): 3064-3074. https://doi.org/10.1016/j.automatica.2012.08.035

[4] Dan, S., Patriciu, A., Petrisor, D., Mazilu, D., Kavoussi, L. (2007). A New Type of Mo-tor: Pneumatic Step Motor. IEEE/ASME Transactions on Mechatronics, 12(1): 98. https://doi.org/10.1109/TMECH.2006.886258

[5] Song, Y., Feng, Y., Ma, J., Zhang, X. (2011). Design of LED Display Control System Based on AT89C52 Single Chip Microcomputer. Journal of Computers, 6(4): 718-724. https://doi.org/10.4304/jep.6.4.718-724

[6] Han, S., Song, J., Zhu, X., Mok, A. K. (2009). Wi-HTest: compliance test suite for di-agnosing devices in real-time WirelessHART ${ }^{\mathrm{TM}}$ mesh networks. Wireless Networks, 21(6): 327-336. https://doi.org/10.1109/RTAS.2009.18

[7] Gungor, V. C., Hancke, G. P. (2009). Industrial Wireless Sensor Networks: Challeng-es, Design Principles, and Technical Approaches. IEEE Transactions on Industrial Electronics, 56(10): 4258-4265. https://doi.org/10.1109/TIE.2009.2015754

[8] Åkerberg, J., Gidlund, M., Lennvall, T., Neander, J., Björkman, M. (2011). Efficient integration of secure and safety critical industrial wireless sensor networks. Eurasip Journal on Wireless Communications \& Networking, 2011(1): 1-13. https://doi.org/10.1186/1687$\underline{1499-2011-100}$

[9] Wang, Y., Wang, W., Yang, W. T. (2008). Control System Design of Acceleration and Deceleration Curves of Stepping Motor and Its Application. Control Engineering of China, 15(5): 576-579.

[10] Bovik, A. C., Maragos, P., Quatieri, T. F. (1993). AM-FM energy detection and separa-tion in noise using multiband energy operators. Signal Processing IEEE Transactions on, 41(12): 3245-3265. https://doi.org/10.1109/78.258071

[11] Xiu, D. B., Sun, Q., Ge, C. Z., Luo, Y., Du, Y. T., Wang, Y. D., Shi, Y. B. (2013). Design of Mobile Meteorological Monitor Based on Wireless Sensor Network. Applied Me-chanics \& Materials, 303-306: 938-944. https://doi.org/10.4028/www.scientific.net/AMM.303$\underline{306.938}$

[12] Wang, L., Li, Y., Fu, G. (2017). Design of ZigBee Wireless Communication Network Node Based on Internet of Things Cloud Platform. Computer Measurement \& Control.

[13] Luo, X., Dong, M. (2013). Distributed Fault-Tolerant Detection in Wireless Sensor Networks. Computers IEEE Transactions on, 55(1): 58-70. https://doi.org/10.1109/TC.2006.13

[14] Banerjee, S., Ghosh, A., Mitra, S. K. (2017). A modified mathematical model for life-time enhancement in wireless sensor network. Mathematical Modelling of Engineer-ing Problems, 4(2): 84-90. https://doi.org/10.18280/mmep.040204 


\section{$7 \quad$ Authors}

Herong Yuan received the master's degree in software engineering from Sichuan University, China in 2014. Currently, she is an experimenter at Institute of electrical and control, Nanjing Polytechnic Institute, Nanjing 210048, China. Her research interests include electrical control and electrical engineering.

Jingbin Song received the Master's degree in application engineering of vehicle from The Northeast forestry university, China in 2007. Currently, he is a adjunct professor at Department of automotive engineering, Changzhou Vocational Institute of Mechatronic Technology, Changzhou 213164, China. His research interests include vehicle inspection and vehicle emission control.

Article submitted 07 August 2018. Resubmitted 23 August 2018. Final acceptance 15 September 2018. Final version published as submitted by the authors. 